\title{
Fulminant Endogenous Anterior Uveitis due to Listeria monocytogenes
}

\author{
Arno Hueber Gerhard Welsandt Rafael S. Grajewski \\ Sigrid Roters \\ Centre of Ophthalmology, University Hospital of Cologne, Cologne, Germany
}

\section{Key Words}

Listeria monocytogenes · Anti-bacterial agents · Drug therapy

\begin{abstract}
Purpose: To report an unusual case of fulminant anterior uveitis, confirmed as endogenous Listeria monocytogenes infection.

Subject: A 67-year-old man with multiple comorbidities acutely developed a severe endogenous anterior uveitis.

Results: L. monocytogenes, a ubiquitous Gram-positive bacillus, was directly indicated by culture and PCR. Early and aggressive treatment with intravenous antibiotics likely prevented the endophthalmitis which most cases on record experienced. Our patient regained satisfactory visual acuity.

Conclusions: Prompt antimicrobial therapy is paramount when severe endogenous uveitis develops in a patient with comorbidities, especially with systemic immunosuppression. Treatment solely with corticosteroids should be avoided.
\end{abstract}

\section{Introduction}

Listeria monocytogenes is a ubiquitous Gram-positive bacillus that rarely incites intraocular inflammation. Only 30 cases of uveitis due to L. monocytogenes have appeared in medical literature since 1967 [1]. The means of entry was hematogenous in each instance.

Cephalosporins are generally ineffective as treatment, but a wide range of antibiotics can be used. Based on expected synergy, a combination of aminopenicillin (amoxicillin or ampicillin) and aminoglycoside is the current treatment of choice [2]. 


\section{Case Report}

A 67-year-old Turkish man presented with deteriorating vision (to hand motion) and pain that increasingly developed in the right eye over a period of 9 days. He was using gentamicin ointment 3 times daily (t.i.d.) for the right eye for 2 days. He had been hospitalized for 17 days with dyspnea, brought on by chronic obstructive pulmonary disease (COPD) and chronic heart failure. This patient also suffered from renal insufficiency and hypertension and had previously undergone aortic valve replacement. Esophagogastroduodenoscopy revealed a Mallory-Weiss syndrome and antral gastritis. Recently, the long-term oral prednisolone therapy he received daily had been increased from 12.5 to 75 $\mathrm{mg}$ because the COPD was getting worse. His ophthalmologic medical history was noncontributory and the left eye was unremarkable. No extraocular septic focus was identifiable.

In the right eye, the conjunctiva was hyperemic, the anterior chamber was visibly inflamed with a 2-mm hypopyon, grayish keratic precipitates, a fixed pupil, and abundant fibrin in front of the lens. Although the posterior segment was not visible, no vitreous involvement was evident in $10 \mathrm{MHz} B$-Scan ultrasound. Intraocular pressure of the right eye was $35 \mathrm{~mm} \mathrm{Hg}$.

Our tentative diagnosis was a bacterial endogenous endophthalmitis with other forms of anterior uveitis as the differential diagnoses. We replaced the existing topical therapy of gentamicin ointment with hourly ofloxacin eye drops, plus night time atropine ointment, and began intravenous delivery of ceftazidime (2 g t.i.d.), tobramycin $(40 \mathrm{mg} / 40 \mathrm{mg} / 80 \mathrm{mg}$ ), and clindamycin (600 mg t.i.d.) in divided daily doses. On day 2, the ofloxacin eye drops were cut to 5 times daily, and we added prednisolone eye drops t.i.d. By day 3, epithelial edema and a 2.5-mm hypopyon were noted. Aqueous humor was then collected by paralimbal paracentesis for microbial study. The hypopyon subsequently dissolved and the intraocular pressure normalised by day 4 .

After 23 days, L. monocytogenes was identified from the aqueous humor sample. Cultures proved positive for Gram-positive rods, confirmed as L. monocytogenes by gene sequencing (16S rRNA). The patient's eye had clearly improved, however, in advance of the diagnosis, so therapy remained unchanged. Four weeks after his referral, both anterior and posterior segment examinations showed no sign of inflammation, except some pigmentary transfer from iris to lens. Given bilateral cataracta provecta, his visual acuity was 0.4 on the right and 0.5 on the left eye.

\section{Discussion}

Patients with multiple comorbidities are often vulnerable to uncommon infections of the eye from ubiquitous, opportunistic bacteria. L. monocytogenes, in particular, may be acquired by humans through direct contact with infected animals, contaminated food (especially raw milk, cheese, ice cream), and unprocessed products of tainted raw meat and fish. In fact, this organism is one of the most frequently transmitted pathogens in food [3]. For bodily dissemination and seeding of sites such as the eye, phagocytosis by phagocytes and hematogenous transport are essential. Intracellular sequestration protects this species from exposure to antibodies and other host defences, thus explaining why all case reports of L. monocytogenes uveitis to date have been endogenous. Onset of gastrointestinal symptoms requires at least 12 hours, while the incubation period for areas elsewhere is several days to weeks.

Previous reports indicate that anterior uveitis of $L$. monocytogenes may be complicated by secondary glaucoma, fibrinous exudate leading to posterior synechiae, and pigmentary precipitates involving the endothelium and anterior lens capsule. Some studies have documented a brown or blackish hypopyon [4], whereas a more characteristic white colour was noted in our case.

Gene sequencing (16S rRNA) facilitates a timely diagnosis [5], but paucity of viable organisms in the culture specimen could easily account for delayed laboratory results. 
This type of uveitis is also typically worsened by standard cortisone therapy, so it is fortunate that we used high-dose intravenous antibiotics from the start. The cephalosporin, ceftazidime, does not penetrate lysosomes and likely had little or no impact [2]. Clindamycin, as our second agent, is a lincosamide antibiotic capable of lysosomal access and is generally effective against this organism [2]. The third antibiotic selected, tobramycin, is an aminoglycoside that is bactericidal with L. monocytogenes and is the first choice, in combination with an aminopenicillin, to treat such infections [2]. Because of early, aggressive antibiotic use, our patient recovered uneventfully and did not develop the endophthalmitis that routinely plagued similar cases depicted in the literature. Various authors share the opinion that early treatment confers the best prognosis with respect to preserving visual acuity [6].

\section{Conclusion}

The above clinical events emphasize the need to consider a bacterial source and promptly initiate antibiotic treatment, whenever a patient of considerable medical complexity, especially with systemic immunosuppression (e.g. oral therapy with corticosteroids), develops endogenous anterior uveitis. Corticosteroids as the sole choice of therapy should be avoided.

\section{Disclosure Statement}

This study was not supported by a grant. The authors have no commercial or proprietary interests in any related products or companies and maintain full control of all primary data.

\section{References}

1 Goodner EK, Okumoto M: Intraocular listeriosis. Am J Ophthalmol 1967;64:682-686.

- Hof H: An update on the medical management of listeriosis. Expert Opin Pharmacother 2004;5:1727-1735.

-3 Ramaswamy V, Cresence VM, Rejitha JS, Lekshmi MU, Dharsana KS, Prasad SP, Vijila HM: Listeria - review of epidemiology and pathogenesis. J Microbiol Immunol Infect 2007;40:4-13.

-4 Eliott D, O’Brien TP, Green WR, Jampel HD, Goldberg MF: Elevated intraocular pressure, pigment dispersion and dark hypopyon in endogenous endophthalmitis from Listeria monocytogenes. Surv Ophthalmol 1992;37:117-124.

5 Lohmann CP, Gabel VP, Heep M, Linde HJ, Reischl U: Listeria monocytogenes-induced endogenous endophthalmitis in an otherwise healthy individual: rapid PCR-diagnosis as the basis for effective treatment. Eur J Ophthalmol 1999;9:53-57.

6 Berger E, Donat M, Guthoff RF, Podbielski A: Listerien-Endophthalmitis [Listeria endophthalmitis]. Ophthalmologe 2005;102:888-890. 